\title{
The effect of national public health measures on the characteristics of trauma presentations to a busy paediatric emergency service in Ireland: a longitudinal observational study
}

\author{
Stephen Gilmartin ${ }^{1}\left[\right.$ - Michael Barrett ${ }^{1,2,3}\left[\right.$ Michael Bennett $^{4} \cdot$ Cliona Begley $^{1} \cdot$ Chantelle Ni Chroinin $^{1}$. \\ Patrick $\mathrm{O}^{\prime}$ Toole $^{5} \cdot$ Carol Blackburn $^{1,2}$
}

Received: 11 December 2020 / Accepted: 7 March 2021 / Published online: 20 March 2021

(c) Royal Academy of Medicine in Ireland 2021

\begin{abstract}
Background The SARS-CoV-2 pandemic and government-enforced restrictions have impacted medical practices.

Aims The aim of our study was to investigate the impact SARS-CoV-2 and public health restrictions had on trauma presentations to a regional paediatric emergency service.

Methods We carried out a multisite retrospective longitudinal study of all paediatric ED attendances from 2018 including 13 March to 7 June 2020. This aligned with the initial government-enforced public health phases: delay phase, mitigation phase and reopening phase 1.

Results There were 7975 total regional attendances during government-enforced restrictions. This represents $17.5 \%$ and $15.6 \%$ reductions in site attendances when compared with the two previous years. Regional attendances reduced by $52.5 \%$ in 2020 compared with 2018 and 50.9\% compared with 2019. Following an initial reduction in injury attendances at the beginning of the 'lockdown' ( $p=0.076)$, the number of injuries consistently grew as weeks progressed $(p<0.05)$, reaching a peak of $44.6 \%$ of all attendances. As restrictions eased, the most common location where injuries occurred moved to areas outside the home $(p<0.000)$. There was a significant change in injury type, final disposition and device-associated injury $(p<0.05)$. Wheeled recreational devices were associated with over $20 \%$ of all injuries by reopening phase 1 .

Conclusions This study reveals that total attendances and total injuries reduced during initial phases of the lockdown. This was followed by a significant increase in injury presentations, which reached a peak of $44.6 \%$ of all attendances. We identified potential modifiable characteristics of paediatric trauma which can be addressed by future public health strategies.
\end{abstract}

Keywords COVID-19 $\cdot$ Injury $\cdot$ Injury prevention $\cdot$ Paediatric emergency medicine $\cdot$ Trauma

Stephen Gilmartin

gilmartinstephen@gmail.com

1 Department of Paediatric Emergency Medicine, Children's Health Ireland Crumlin, Dublin, Ireland

2 School of Medicine, Women's and Children's Health, University College Dublin, Dublin, Ireland

3 National Children's Research Centre, Crumlin, Dublin, Ireland

4 Department of Paediatric Emergency Medicine, Children's Health Ireland Tallaght, Dublin, Ireland

5 Department of Trauma and Orthopaedics, Children's Health Ireland, Dublin, Ireland

\section{Introduction}

The severe acute respiratory syndrome coronavirus 2 (SARS-CoV-2) pandemic has impacted all areas of modern medical practice [1,2]. On 11 March when the World Health Organization announced that the outbreak was now a global pandemic, the Irish government began to enforce strict measures to curb the impact of the virus. As of 13 March, these measures included closure of schools, prohibition of large gatherings and the cessation of organised sporting events. These restrictions limited many of the activities which carry a high risk of paediatric injury $[3,4]$.

In Ireland a 30\% reduction in emergency department (ED) attendances was observed when compared with the same period in 2019, and similar reductions have been experienced internationally [5-7]. Using national health authority 
administration data, a study from Ireland shows a reduction in the proportion of all patients presenting at ED across all acuity presentations [8]. This phenomenon extended to paediatric EDs with one Irish study showing a $>50 \%$ reduction in total attendances compared with 2019 [9].

In normal circumstances, a seasonal variation in paediatric injury-related presentations exists $[10,11]$. The annual upsurge in paediatric injuries during the summer months is largely attributable to good weather conditions and increased daylight hours, leading to unsupervised outdoor activity for longer periods. Although the SARS-CoV-2 pandemic is unprecedented in modern literature, prior studies performed during the Middle Eastern respiratory syndrome (MERS) and severe acute respiratory syndrome (SARS) epidemics $[12,13]$ show a significant decrease in paediatric emergency department presentations but an increase in injury rates [13].

Children's Health Ireland Crumlin (CHI at Crumlin) is the largest tertiary paediatric hospital in Ireland and, due to the COVID-19 paediatric service reconfiguration, became the largest paediatric ED [14]. Like every hospital, the institution had to implement changes to its emergency department footprint and layout and to hospital services in general in preparation for a pandemic. The regional approach is unlike those of other responding hospitals across Europe as identified by a study investigating the impact of the coronavirus (COVID-19) pandemic on children presenting to emergency departments across Europe [15]. In late March 2020, CHI Crumlin ED had to manage changes in attendance patterns brought about by the temporary closure of paediatric ED and inpatient services provided by a sister paediatric hospital with a paediatric emergency department (PED) (7 km away) which closed to facilitate surging adult COVID-19 presentations at that site [16].

The aim of our study was to analyse the impact SARSCoV-2 and government-enforced restrictions had on injury presentations to our paediatric ED. The authors hope this information will inform future strategic steps needed to better meet the needs of paediatric EDs as the pandemic continues.

\section{Methods}

Setting $\mathrm{CHI}$ Crumlin and $\mathrm{CHI}$ at Tallaght are a regional service with a national referral tertiary multi-universityaffiliated institution and secondary paediatric hospital with on-site paediatric EDs at both sites. The two paediatric EDs provide the only public sites of regional emergent paediatric care. This region encompasses at least Wicklow, East Kildare, Dublin West, South City, South and South West with a population of over 1 million. The combined ED census was 75,000 patients in 2019. Due to regional COVID-19 service reconfiguration, $\mathrm{CHI}$ at Crumlin became the single point of access for regional emergent paediatric care on 27 March 2020.

Data and design We carried out a retrospective longitudinal multisite regional study of ED attendances from 13 March to 7 June 2020. This period coincided with the delay phase, mitigation phase and reopening phase 1 of the government's public health response (Fig. 1). The mitigation phase was the period of most stringent restrictions, and reopening phase 1 represents when these restrictions began to ease. We collected attendance data from matched periods in 2019 and 2018 for comparison.

Patients attending with musculoskeletal injuries, wounds, burns and head injuries were identified through a search of the electronic patient management system Symphony. Source data was derived from Symphony®, the national integrated medical imaging service NIMIS, patient records and prehospital records. Data points included date of birth, days since injury, location where injury was sustained, major trauma presentations, sport-related injuries, device-related injuries and final diagnosis. All records were pseudo-anonymised; data was stored and analysed using Microsoft Excel®.

Total regional attendances and numbers of injury presentations during matched periods in 2019 and 2018 were collected for comparison. This included total attendance and injury attendance figures to our sister PED from 28 March 2018 and 2019. The date 28 March was chosen as our sister PED closed at midnight on 27 March 2020 as part of regional COVID-19 service reconfigurations.

Data was expressed as mean and standard deviations for the quantitative variables and as numbers and percentages for categorical variables. Descriptive statistical analysis was performed using the $\mathrm{R}{ }^{\circledR}$ statistical computing software. Mean weekly counts for injury presentations between successive phases were compared using the Mann-Whitney $U$ test. Proportions between successive phases were compared using the chi-squared test. A quasi-Poisson regression was

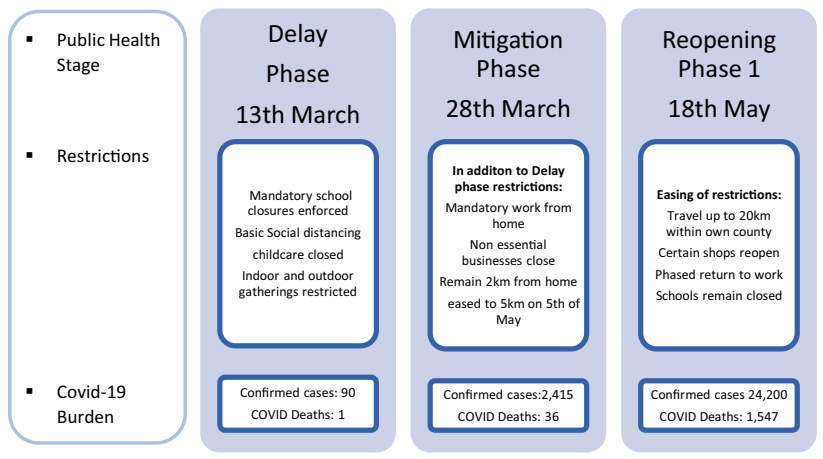

Fig. 1 Stages of government public health response 
used to compare week-to-week variation in injury presentations within phases. Statistical significance was defined as $p<.05$.

Institutional ethics approval was sought and obtained from the Research Ethics Committee at Children's Health Ireland Crumlin.

\section{Results}

\section{Total attendances}

The paediatric ED census was 7975 attendances from 13 March to 7 June 2020. This is compared with 9670 and 9457 attendances over a similar period in 2018 and 2019 respectively. This represents $17.5 \%$ and $15.6 \%$ reductions in CHI Crumlin ED census when compared with the two previous years. With addition of attendance figures from our sister PED, there was 16,820 combined regional attendances in 2018 and 16,236 in 2019 for the time periods of interest. This is a $52.5 \%$ reduction in regional ED census in 2020 compared with 2018 and a $50.9 \%$ reduction compared with 2019.

It is apparent from Fig. 2 that during the delay phase of government restrictions, there were considerable reductions in overall attendances. These figures did trend upwards as phases progressed but never fully recovered.

\section{Injuries}

A total of 2864 injury presentations were identified and reviewed during the study period. In Fig. 3, injury-related presentations are plotted against the same dates in 2018

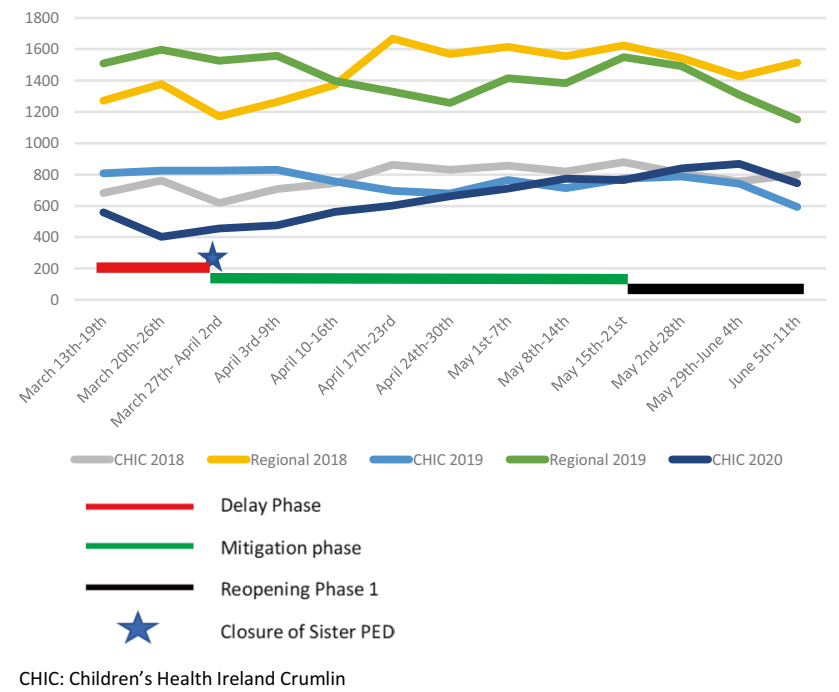

Fig. 2 Total attendance trends: 2020 vs 2018 and 2019

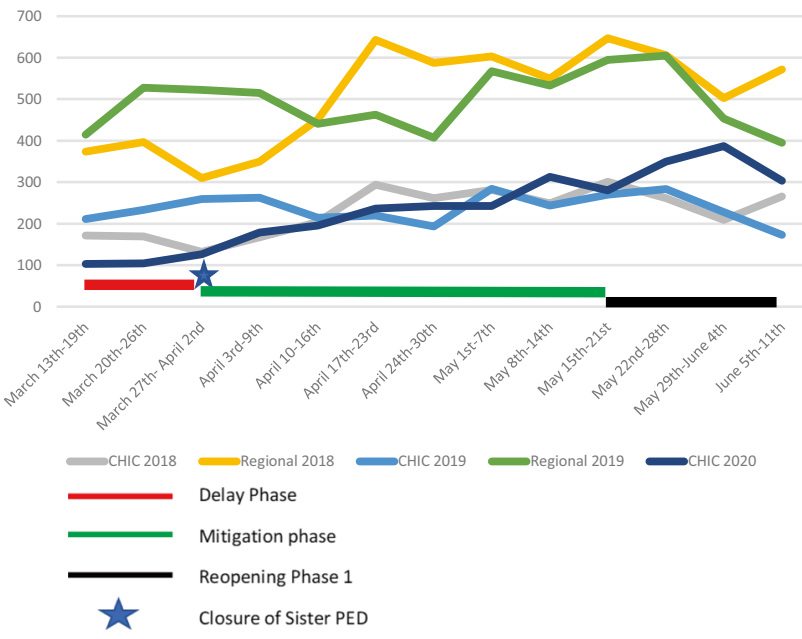

CHIC: Children's Health Ireland Crumlin

Fig. 3 Total injuries: 2020 vs 2018 and 2019

and 2019. In CHI Crumlin ED, there were 2852 injuryrelated presentations in 2018 and 2976 in 2019. Combined with our sister PED, there were 5849 in 2018 and 5666 in 2019. A Mann-Whitney $U$ test was used to compare phase-to-phase progression of total injury presentations in 2020. There was a significant increase between the delay phase and mitigation phase $(p=0.012)$; a similar rise was seen when comparing the mitigation phase to reopening phase $(p=0.016)$. A quasi-Poisson regression was used to compare variation from week to week within phases. A reduction in total injury attendances was seen in the delay phase, but this was not statistically significant $(p=0.076)$. There was a significant increase in total injury presentations during the mitigation and reopening phases. Of note in Figs. 2 and 3, the final time period 5-7 June did not represent a full week. An average daily attendance was used to estimate total attendances and total injuries for this period to allow comparison.

Injuries represented $35.91 \%$ of all presentations. During week $1,18.5 \%$ of attendances were injury related and a peak of $44.6 \%$ was seen in week 12 . Figure 4 displays how these figures compare with 2018 and 2019. Injuries represented 29.49\% and $31.47 \%$ of all attendances in CHI Crumlin ED during 2018 and 2019 respectively. In combined regional ED figures from 2018 and 2019, injuries represented 34.7\% of attendances for 2018 and $34.9 \%$ for 2019.

A change in location where injuries occurred was noted as restrictions changed and time progressed as displayed in Table $1(p<0.000)$. During the delay phase, $80.47 \%$ of all injuries occurred at home either inside or outside. During the mitigation phase, this had reduced to $75.32 \%$, and during reopening phase 1 , only $56.20 \%$ of injuries occurred at home. There is an inverse rise in the number of cases per week in nearly all other major categories as reopening 


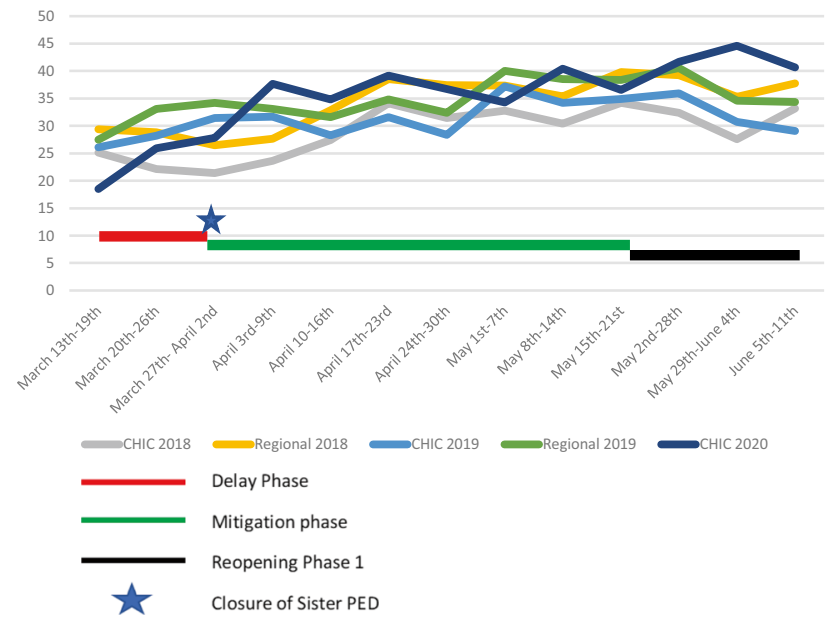

CHIC: Children's Health Ireland Crumlin

Fig. 4 Injuries as a percentage of total attendances: 2020 vs 2018 and 2019

occurs. This is in line with the previously observed overall increase in injury presentations.

Looking at devices involved in injury presentations, a significant change in presentation patterns as phases progress was again observed $(p<0.000)$. Wheeled non-motorised vehicles were the most common devices contributing to injury. This included bicycles, scooters, skateboards and rollerblades. Trampolines were consistently the second most common. There was a progressive increase in injuries from wheeled, non-motorised vehicles as we moved through the phases with a high of $63.85 \%$ of the device-related injuries and $21.56 \%$ of all injuries during reopening phase 1 .

The government-enforced restrictions prevented organised sport participation and included a ban on large outdoor gatherings. As a result, injuries occurring during sport did not represent a significant group during any phase. Injuries attributable to organised sports noted during the mitigation phase and reopening phase 1 were due to horse riding alone.

In Table 2 we see a significant change in diagnoses made was observed as phases of restriction progressed $(p<0.000)$. Fractures represented $26.51 \%$ of injuries during the delay phase; this figure had risen to $30.51 \%$ of injuries during phase 1 of reopening. A converse reduction in the proportion of burns, head injuries and fingertip injuries was also observed. There was no change in major trauma presentations. Major trauma presentations remained $<2 \%$ of all injuries during all phases.

Over $60 \%$ of patients were discharged without any requirement for further follow-up. The orthopaedic service runs a virtual trauma assessment clinic which manages follow-up for uncomplicated fractures. This involves a multidisciplinary team who review the relevant radiographs and patient notes prior to conducting a telephone call with the patient's carers. This prevents the need for further patient attendance to hospital. The virtual clinic provided care for $17.67 \%$ of injured patients during the delay phase; this fell to $15.49 \%$ during the mitigation phase $(-2.18 \%)$ and $14.37 \%$ during reopening phase $1(-3.3 \%)$. There was a corresponding rise in the proportion of patients seen in a 'face-to-face' fracture clinic from a low of $6.05 \%$ during the delay phase to $8.02 \%(+1.97 \%)$ during the mitigation phase and $11.61 \%$ $(+5.56 \%)$ in reopening phase 1 . This change in patient disposition as phases progressed was once again found to be statistically significant $(p=0.03)$.

\section{Discussion}

This study presents the profile of regional trauma presentations during the initial public health-recommended, government-imposed management stages of the current COVID-19 pandemic. Injury presentations surpassed a single department's figures from the previous 2 years by the end of the delay phase. This was contributed to by the closure of a sister regional PED. When we look at the total attendances and injury presentations from our own department and our sister PED, we see that attendances due to injury remain significantly reduced compared with the two previous years. From the 4th week of the delay phase, the percentage of total presentations represented by injury showed a consistent upward trend and reached a peak of $44.6 \%$ of all presentations in week 12 of restrictions (week 3 of the reopening phase). This is an unprecedented figure with almost one in two attendances related to injury. A similar pattern was noted during the MERS epidemic [13] when the proportion of paediatric injury presentations increased while overall paediatric attendances decreased.

Seasonal variations in paediatric injuries are well documented [17], and it appears that during government public health restrictions, this seasonal spike was moved forward 2 months to the start of April. This is despite the Irish government's public health guidance which imposed domestic lockdown, closure of playgrounds and cessation of organised sport. The observed, higher than usual proportion of injuries which attended the PED was likely significantly contributed to by the rapid termination of the winter viral season resulting from severe restrictions limiting both adults and children to home.

The largest burden of injury was associated with wheeled non-motorised vehicles (WNMV). WNMV represented nearly two-thirds of all device-related injuries during this study period and increased greater than fivefold between initial and reopening phases. The lack of WNMV usage during the delay phase when children were restricted to their homes likely contributed to the smaller number of injury-related presentations. This is a potentially modifiable cause of 


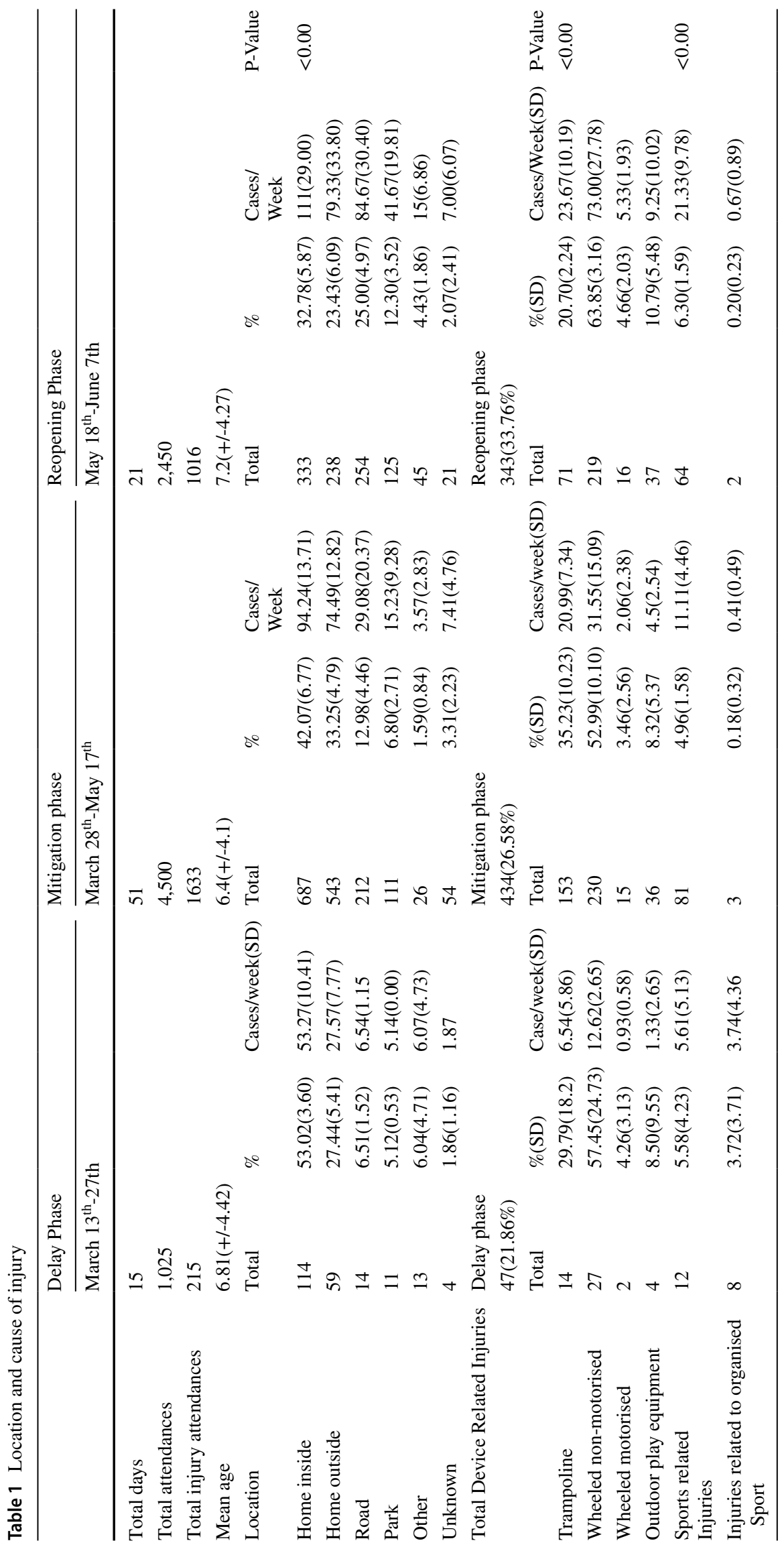


Table 2 Diagnosis and patient disposition

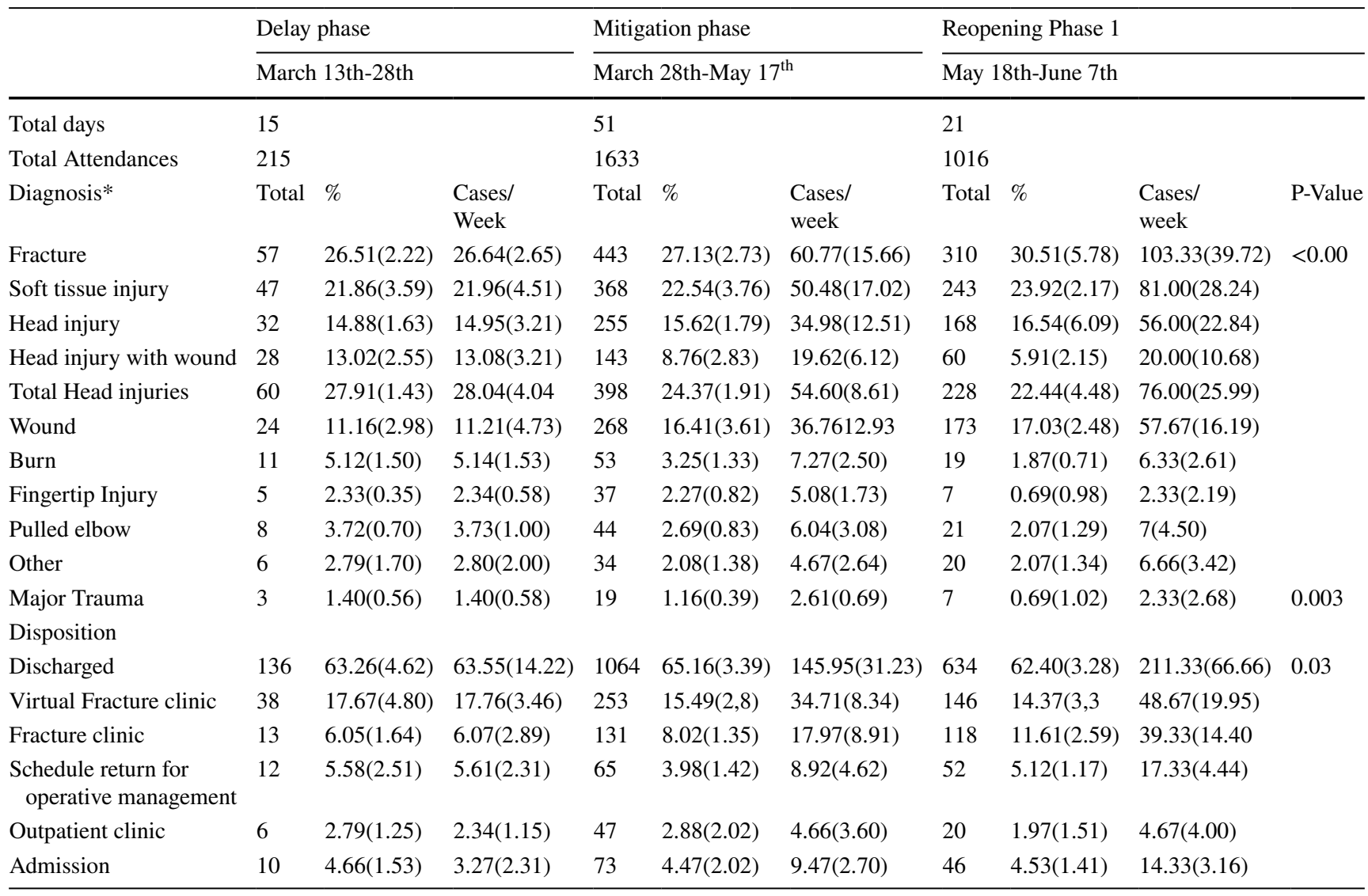

*Percentages to not add up to $100 \%$ as some patients suffered multiple injuries

injury. Research has shown the positive impact simple interventions such as helmet use can have on WNMV-associated injuries $[18,19]$. Currently, Ireland has no injury prevention/ safety legislation related to these vehicles. We hypothesise a reduction in WNMV injury burden could occur if public health interventions (e.g. awareness of safety) or legislation (e.g. mandating safety equipment) addressed modifiable characteristics of paediatric trauma similar to other jurisdictions [20].

Another key finding of this study was the significant change in the location where injuries were sustained $(p<0.000)$, as phases progressed. This is expected considering the public health restriction measures employed. During the delay phase, $80.47 \%$ of all injuries occurred at home. During the mitigation phase, this reduced to $75.32 \%$, and it reduced further to $56.2 \%$ during reopening phase 1 when people were once again allowed to gather in groups outside and playgrounds reopened. These patterns can be attributed to relaxation of restrictions, but passage of time and 'restriction fatigue' may also have been contributing factors.

Interestingly, during the delay phase, most patients with fractures were suitable for outpatient management via the virtual fracture clinic and did not require a face-to-face fracture clinic visit (17.67\% vs $6.05 \%)$. As the phases progressed, more patients required face-to-face fracture clinic follow-up. During the reopening phase, $11.61 \%$ attended a face-to-face fracture clinic and $14.37 \%$ were assessed in the virtual clinic. This likely reflects an increase in the number of children experiencing more significant or complex fractures as restrictions eased.

This study has limitations. Firstly, it was conducted as a retrospective review. Data collection was triangulated from multiple primary sources of data including electronic information systems, ambulance record, triage notes, radiological ordering systems and hard copy patient records. Our sister hospital closed its paediatric emergency department on 27 March 2020 due to COVID-19-related capacity requirements in the co-located adult facility, and we cannot account for subsequent emergency paediatric care delivered outside the region. Despite the increase in catchment population, total attendances remained less than 2018 and 2019 for much of the study period. This effect should not affect the characteristics of presenting injuries regionally.

In conclusion, this study has shown that in the early phases of restrictions, total attendances and total injuries reduced substantially. This was followed by a significant 
increase in injury presentations, which reached a peak of $44.6 \%$ of all presentations. As the restrictions eased, the most common location of injury occurrence moved outside the home and a quarter were associated with roads by reopening phase 1 . Interestingly, wheeled recreational devices were associated with over $20 \%$ of all injuries by reopening phase 1 and is an opportunity for future targeted public health initiatives or legislation to improve child safety/injury prevention.

Acknowledgements The authors would like to acknowledge the exceptional help Jennifer Sheedy provided with the statistical analysis.

Author contribution SG, CB and P O'T designed the study protocol. $\mathrm{SG}, \mathrm{MBe}, \mathrm{Cl} \mathrm{B}$ and $\mathrm{C}$ Ni C collected data. MB and SG conducted statistical analysis. SG wrote the initial manuscript, and all authors contributed revisions. All authors take responsibility for the findings of the study.

Data availability Data is available for distribution following reasonable request.

\section{Declarations}

Conflict of interest The authors declare that they have no conflicts of interest.

\section{References}

1. McAuliffe E, Hamza M, McDonnell T et al (2020) Children's unscheduled primary and emergency care in Ireland: a multimethod approach to understanding decision making, trends, outcomes and parental perspectives (CUPID): project protocol. BMJ Open. 10(8):e036729

2. Nicholson E, Mcdonnell T, Conlon C et al (2020) Understanding the impact of public health advice on paediatric healthcare use during COVID-19: a cross-sectional survey of parents

3. Trefan L, Houston R, Pearson G et al (2016) Epidemiology of children with head injury: a national overview. Arch Dis Child [Internet]. 101(6):527 LP - 532. Available from: http://adc.bmj.com/ content/101/6/527.abstract

4. Beck B, Teague W, Cameron P, Gabbe BJ (2019) Causes and characteristics of injury in paediatric major trauma and trends over time. Arch Dis Child [Internet]. 104(3):256 LP - 261. Available from: http://adc.bmj.com/content/104/3/256.abstract

5. Stephen M(2020) Non Covid19 patients and service users (IPA STATEMENT) [Internet]. Available from: https://www.irishpatients.ie/post/ non-covid19-patients-and-service-users-ipa-statement

6. Thornton J (2020) Covid-19: A\&E visits in England fall by $25 \%$ in week after lockdown. BMJ [Internet]. 369:m1401. Available from: http://www.bmj.com/content/369/bmj.m1401.abstract

7. IAEM (2020) The current CoVid19 impact on Emergency Departments [Internet]. Available from: http://www.iaem.ie/wp-content/ uploads/2020/03/IAEM-PR-on-the-current-impact-of-CoVid-19on-Emergency-Departments-200320.pdf
8. Brick A, Walsh B, Keegan C et al (2020) COVID-19 and emergency department attendances in public hospitals. ESRI Special Article

9. Dann L, Fitzsimons J, Gorman KM et al (2020) Disappearing act: COVID-19 and paediatric emergency department attendances. Arch Dis Child [Internet]. Archdischild-2020-319654. Available from: http://adc.bmj.com/content/early/2020/06/09/archdischild-2020319654.abstract

10. Jespersen E, Holst R, Franz C et al (2014) Seasonal variation in musculoskeletal extremity injuries in school children aged 6-12 followed prospectively over 2.5 years: a cohort study. BMJ Open [Internet]. 4(1):e004165. Available from: http://bmjopen.bmj. com/content/4/1/e004165.abstract

11. Foltran F, Avossa F, Fedeli U et al (2013) Seasonal variations in injury rates in children: evidence from a 10 -year study in the Veneto Region, Italy. Int J Inj Contr Saf Promot [Internet]. 20(3):254-8. Available from: https://doi.org/10.1080/17457300.2012.69269

12. Man C, Yeung R, Chung J, Cameron P (2003) Impact of SARS on an emergency department in Hong Kong. Emerg Med (Fremantle). 1(15):418-22

13. Paek SH, Kim DK, Lee JH, Kwak YH (2017) The Impact of Middle East respiratory syndrome outbreak on trends in emergency department utilization patterns. J Korean Med Sci [Internet]. 32(10):157680. Available from: https://pubmed.ncbi.nlm.nih.gov/28875599

14. HSE (2020) CHI advise of temporary relocation of acute paediatric services at $\mathrm{CHI}$ at Tallaght [Internet]. 2020. Available from: https://www.hse.ie/eng/services/news/media/pressrel/chi-adviseof-temporary-relocation-of-acute-paediatric-services-at-chi-attallaght.html

15. Nijman R (2020) Investigating the impact of the coronavirus (COVID-19) pandemic on children presenting to emergency departments across Europe. Available from: https://doi.org/10.1186/ ISRCTN91495258

16. Bressan S, Buonsenso D, Farrugia R et al (2020) Preparedness and response to Pediatric CoVID-19 in European Emergency Departments: a survey of the REPEM and PERUKI networks. Ann Emerg Med [Internet]. Available from: http://europepmc.org/abstract/MED/ 32419713

17. Segal D, Slevin O, Aliev E et al (2018) Trends in the seasonal variation of paediatric fractures. J Child Orthop [Internet]. 12(6):614-21. Available from: https://pubmed.ncbi.nlm.nih.gov/ 30607209

18. Lindsay H, Brussoni M (2014) Injuries and helmet use related to non-motorized wheeled activities among pediatric patients. Chronic Dis Inj Can. 1(34):74-81

19. Bressan S, Daverio M, Barker R et al (2016) Paediatric recreational vehicle-related head injuries presenting to the emergency department of a major paediatric trauma centre in Australia: is there room for improvement? Emerg Med Australas [Internet]. 28(4):425-33. Available from: https://doi.org/10.1111/1742-6723.12617

20. Government Q (2018) Safety Rules: Skateboards, foot scooters and similar [Internet]. Available from: https://www.qld.gov.au/ transport/safety/rules/wheeled-devices/skateboards

Publisher's Note Springer Nature remains neutral with regard to jurisdictional claims in published maps and institutional affiliations. 